\title{
Publisher Correction: Control of semiconductor emitter frequency by increasing polariton momenta
}

Yaniv Kurman, Nicholas Rivera, Thomas Christensen, Shai Tsesses, Meir Orenstein, Marin Soljačić, John D. Joannopoulos and Ido Kaminer

Correction to: Nature Photonics https://doi.org/10.1038/s41566-018-0176-6, published online 4 June 2018.

In the version of this Article originally published, in the PDF, in equations (1) and (3b), the superscript ' $\rho$ ' was mistakenly set as ' $\mathbf{j}$ '; the equations should have read:

$$
\begin{gathered}
\mathbf{E}_{\mathbf{q}}(\mathbf{r}) \propto \mathrm{e}^{-q|z|} \mathrm{e}^{-i \mathbf{q} \cdot \rho}(\hat{\mathbf{q}} \pm i \hat{\mathbf{z}}) \\
\psi_{n \mathbf{k}}(\mathbf{r})=\mathrm{e}^{-i \mathbf{k} \cdot \rho} \phi_{n}\left(z-z_{0}\right)
\end{gathered}
$$

The equation number (5) was missing from the following equation:

$$
\begin{aligned}
\underset{\mathrm{ab}}{\Gamma \mathrm{em}}= & \frac{2 \pi}{\hbar} \sum_{\mathbf{k}_{\mathrm{i}} \mathbf{k}_{\mathrm{f}}}\left|\left\langle n_{\mathrm{f}}, \mathbf{k}_{\mathrm{f}}, n_{\mathbf{q}} \pm 1\left|H_{\mathrm{int}}\right| n_{\mathrm{i}}, \mathbf{k}_{\mathrm{i}}, n_{\mathbf{q}}\right\rangle\right|^{2} \\
& f\left(\mathbf{k}_{\mathrm{i}}\right) \delta\left(E_{n_{\mathrm{i}} \mathbf{k}_{\mathrm{i}}}-E_{n_{\mathrm{f}} \mathbf{k}_{\mathrm{f}}} \mp \hbar \omega_{q}\right)
\end{aligned}
$$

In equation (6), the proportional sign ' $\alpha$ ' was mistakenly included in term (i), the equation should have read:

$$
\begin{aligned}
& \left\langle n_{\mathrm{f}}, \mathbf{k}_{\mathrm{f}}, n_{\mathbf{q}} \pm 1\left|H_{\mathrm{int}}\right| n_{\mathrm{i}}, \mathbf{k}_{\mathrm{i}}, n_{\mathbf{q}}\right\rangle \propto \\
& \overbrace{\left\langle n_{\mathrm{f}}\left|\mathrm{e}^{-q z} \partial_{z}\right| n_{\mathrm{i}}\right\rangle}^{\text {(i) }} \overbrace{\left\langle\mathbf{k}_{\mathrm{f}}\left|\mathrm{e}^{ \pm i \mathbf{q} \cdot \rho}\right| \mathbf{k}_{\mathrm{i}}\right\rangle}^{\text {(ii) }}
\end{aligned}
$$

And, in both the PDF and HTML versions, in the equation in the sentence beginning "The results presented are normalized to yield...", the denominator ' $\pi^{2}$ ' was incorrect; it should have been ' $(2 \pi)^{2}$ '. These errors have now been corrected online. 